\title{
BMJ Open Multifaceted intervention to enhance cognition in older people at risk of cognitive decline: study protocol for the Protein Omega-3 and Vitamin D Exercise Research (PONDER) study
}

\author{
Helen Macpherson, ${ }^{0}$ Sarah Brownell, ${ }^{1}$ Rachel L Duckham, ${ }^{1,2}$ Barbara Meyer, ${ }^{3}$ \\ Sam Mirzaee, ${ }^{4}$ Robin M Daly ${ }^{1}$
}

To cite: Macpherson $\mathrm{H}$, Brownell S, Duckham RL, et al. Multifaceted intervention to enhance cognition in older people at risk of cognitive decline: study protocol for the Protein Omega-3 and Vitamin D Exercise Research (PONDER) study. BMJ Open 2019;9:e024145. doi:10.1136/ bmjopen-2018-024145

- Prepublication history for this paper is available online. To view these files, please visit the journal online (http://dx.doi. org/10.1136/bmjopen-2018024145).

Received 18 May 2018 Revised 19 December 2018 Accepted 12 March 2019
D) Check for updates

(C) Author(s) (or their employer(s)) 2019. Re-use permitted under CC BY-NC. No commercial re-use. See rights and permissions. Published by BMJ.

For numbered affiliations see end of article.

\section{Correspondence to} Dr Helen Macpherson; helen.macpherson@deakin. edu.au

\section{ABSTRACT}

Introduction An increasing number of people are living with cognitive impairment and dementia. Current pharmacological therapies at best reduce Alzheimer's disease symptomatology but do not delay dementia onset in those at high risk. Structured exercise interventions can enhance cognition in older people; however, to produce long lasting, clinically relevant cognitive benefits, it is proposed that a multifaceted approach incorporating exercise with dietary supplements will address a wider range of mechanisms involved in cognitive decline. The Protein Omega-3 and vitamin D Exercise Research (PONDER) study aims to investigate the cognitive effects of a multimodal exercise programme combined with nutritional supplementation in older adults with subjective memory impairment (SMI).

Methods and analysis The PONDER study is a singlecentre, 12-month, community-based, parallel group, randomised, double-blind, placebo controlled trial involving a 6-month multifaceted intervention with a further 6-month follow-up. Participants will be 148 people from Melbourne, Australia, aged 60-85 years with SMI who will be randomised (1:1 ratio) to either a 6 -month supervised multimodal exercise programme combined with omega-3 fatty acid, vitamin $D$ and protein supplementation or a stretching/flexibility exercise programme combined with placebo supplements. The primary outcome is the change in cognition after 6 months as assessed by the Trail Making Test and global cognitive function assessed from the Cogstate Computerised battery. Secondary outcomes will include memory, working memory/learning and attention/ psychomotor function, the Montreal Cognitive Assessment, mood, quality of life, muscle strength, physical function, body composition, cardiovascular health and sleep quality. Cognition at 12 months will represent a secondary outcome.

Ethics and dissemination This study has been approved by the Deakin University Human Research Ethics Committee (project 2016-260). Informed consent will be obtained from all participants. The authors intend to submit the findings of the study to peer-reviewed journals or academic conferences to be published.

Trial registration number ACTRN12616001549415; Preresults.

\section{Strengths and limitations of this study}

- This is the first randomised controlled trial to investigate the combined effects of a multimodal exercise programme with omega- 3 , vitamin $D$ and protein supplementation on cognition in older adults with subjective memory impairments.

- The exercise programme is structured, supervised and delivered in a community, rather than a laboratory setting.

- The study has an additional follow-up period to enable assessment of longer term cognitive changes (over 12 months).

- It was not feasible to examine the isolated effects of the exercise programme and dietary supplement in separate study arms.

\section{INTRODUCTION}

Globally, the population is ageing, and the number of people living with dementia is set to increase by at least $50 \%$ in the next 20 years. ${ }^{1}$ Delaying dementia onset by as little as 6 months could reduce prevalence by $6 \%$, with a delay of 5 years predicted to decrease prevalence by $44 \%{ }^{2}$ Therefore, strategies to reduce dementia risk factors, delay dementia onset and to slow the rate of neuropathology are urgently needed to reduce the economic and social burden of dementia and related cognitive disorders.

Alzheimer's disease (AD) is a form of dementia characterised by progressive neurodegeneration, decline in memory and other cognitive processes. Current pharmacological treatments at best reduce $\mathrm{AD}$ symptomatology and have not demonstrated efficacy to delay dementia onset in those at high risk of developing AD. ${ }^{3}$ Mechanisms contributing to the neuropathology of $\mathrm{AD}$ are known to include neuroinflammation, oxidative stress, 
mitochondrial and cerebrovascular dysfunction. ${ }^{4}$ Interventions that target such modifiable processes, while promoting neuroplasticity and neuroprotection are essential to combat cognitive decline and $\mathrm{AD}$.

\section{Exercise, omega-3 fatty acids and vitamin D for cognitive function}

Exercise is a candidate to attenuate neuropathology and enhance brain health throughout life via the regulation of growth factors and neurotrophins such as insulin-like growth-factor 1 (IGF-1), brain-derived neurotrophic factor (BDNF) and vascular endothelial growth factor that are involved in angiogenesis, synaptogenesis and neurogenesis and the modulation of systemic inflammation. ${ }^{5}$ Exercise can positively influence cardiovascular health and insulin resistance, which are pathways relevant to cognitive decline. ${ }^{6}$ A large body of research has demonstrated that greater levels of physical activity are associated with slower rates of cognitive decline ${ }^{7}$ and a lower risk of dementia. ${ }^{8}$ Furthermore, randomised controlled trials (RCTs) have shown that even acute bouts of exercise can provide small benefits to cognition. ${ }^{9}$ Findings from recent meta-analyses have indicated that longer duration exercise interventions can enhance cognition in healthy p eople $>50$ years of age ${ }^{10}$ as well as individuals at risk of or diagnosed with $\mathrm{AD} .{ }^{11}$

While questions still remain regarding the optimal mode and dose of exercise required for cognitive improvements, ${ }^{12}$ a recent systematic review and meta-analysis of 36 RCTs in community-dwelling adults, aged 50 years and above, ${ }^{10}$ provided the recommendation that combined aerobic and resistance exercise, at a moderate to high intensity, as many days of the week as feasible, is suitable to enhance cognitive function. Exercise interventions of 4-6 months' duration that have used resistance or multimodal programmes, incorporating resistance training, have indicated that, in older people, a frequency of two sessions per week can benefit cognition. ${ }^{13-15}$ Importantly, cognitive benefits have been observed for resistance training when compared with 'sham' exercise or stretching conditions designed not to impact heart rate or aerobic capacity. ${ }^{13} 1416$ The use of a control exercise condition that is matched for social interaction, including attention from the trainer delivering the programme, is necessary to account for possible benefits to cognition attributed to the social experience, rather than the exercise programme.

Accumulating evidence, largely from epidemiological studies, suggests that various dietary factors alone and in combination, particularly omega- 3 fatty acids and vitamin $\mathrm{D}$, are strongly linked with cognitive decline and dementia. ${ }^{17-20}$ Polyunsaturated fatty acids (PUFAs) serve an essential role in the brain, regulating the function and structure of neurons, endothelial cells and glial cells. ${ }^{21}$ The long-chain omega-3 fatty acids eicosapentaenoic acid (EPA) and docosahexaenoic acid (DHA) also modify neurotransmission, reduce neuroinflammation and promote neuronal survival and neurogenesis. ${ }^{21}$ The high intake of omega- 6 fatty acids in the Western diet (ie, from vegetable oil) causes an imbalance of omega$3 / 6$ fatty acids, resulting in lower than optimal levels of DHA and EPA. ${ }^{22}{ }^{23}$ A high intake of omega- 6 fatty acids has been suggested to adversely impact cognition by depleting DHA, the omega-3 PUFA most prevalent in the brain. ${ }^{24}$ In contrast, a low omega- $6 / 3$ ratio has been shown to predict better cognitive function in healthy older people. ${ }^{25}$

Greater dietary intake of DHA (from consumption of fish) has been linked to better cognitive outcomes in older people ${ }^{26}$ and a $36 \%$ lower risk of $\mathrm{AD}$ across multiple observational studies. ${ }^{17}$ A dose-response meta-analysis showed that an increment of $100 \mathrm{~g}$ per week of fish intake was associated with an $11 \%$ lower risk of $\mathrm{AD} .{ }^{17}$ In older people with mild memory complaints $(\mathrm{n}=437) 900 \mathrm{mg} /$ day of DHA supplementation for 24 weeks improved episodic memory and learning on computerised measures. ${ }^{27}$ Of note, when compared with normative data, the improvements due to DHA supplementation on the verbal learning test approximated a 7-year improvement, compared with a 3.6-year improvement with placebo. Another study conducted in 50 older people with mild cognitive impairment found that both EPA rich (1.67g EPA $+0.16 \mathrm{~g}$ DHA/ day) and DHA rich (1.55 g DHA+0.40 g EPA/day) supplements, taken daily for 6 months, improved depressive symptoms, compared with an omega-6 rich supplement. ${ }^{28}$ In this study, only the DHA-rich supplement improved a single cognitive domain as well as physical health. As not all studies have yielded positive results regarding omega-3 fatty acid supplementation on cognitive function, ${ }^{29-31}$ dose and target population may be crucial factors.

An emerging role for vitamin $\mathrm{D}$ in the maintenance of brain health and cognition is developing with reports that serum 25-hydroxyvitamin D $(25(\mathrm{OH}) \mathrm{D})$ levels are lower in those with impaired cognitive function and $\mathrm{AD}$ than healthy controls ${ }^{182}$ and low vitamin D levels increase AD risk 7 years later. ${ }^{33}$ Observational studies indicate that inadequate vitamin $\mathrm{D}$ intake is linked to poorer cognitive function ${ }^{34}$ and may increase the probability of cognitive impairment by as much as $30 \%{ }^{35}$ Relatively few RCTs have investigated the impact of vitamin $\mathrm{D}$ supplementation on cognition in older adults, ${ }^{35}$ although clinical trial registries indicate a number of studies are underway. In a recent 18-week study comparing high-dose vitamin D3 (4000 IU/day) to a low dose (400 IU/day) in 82 healthy adults, the high-dose supplementation improved visuospatial memory but not other cognitive domains. ${ }^{36}$ As even the low dose increased $25(\mathrm{OH}) \mathrm{D}$ concentrations above $75 \mathrm{nmol} / \mathrm{L}$, this may have impacted the ability to detect cognitive changes dues to higher dose supplementation, as $75 \mathrm{nmol} / \mathrm{L}$ is the suggested optimum value to exceed to reduce cognitive and brain dysfunction. ${ }^{37}$

Whether there is an additive effect of different nutrients with exercise in terms of benefits to brain health is an important question. Research in rodents has indicated that DHA can enhance the effects of exercise on BDNF-related synaptic plasticity, ${ }^{38}$ and vitamin $\mathrm{D}$ has 
the potential to contribute to these actions through the regulation of neurotrophins BDNF, glial cell line-derived neurotrophic factor, nerve growth factor and IGF-1. ${ }^{35}$ As a neuroprotective agent, vitamin $\mathrm{D}$ may target specific $\mathrm{AD}$ pathology, attenuating amyloid-beta $(\mathrm{A} \beta)$ accumulation by stimulating the phagocytosis of the $A \beta$ peptide and inducing cytokines and macrophages to increase $A \beta$ clearance. ${ }^{35}$ Exercise and DHA exert potent anti-inflammatory effects ${ }^{521}$ and vitamin D may further attenuate inflammation-induced degeneration of neurons by inhibiting the production of tumour necrosis factor- $\alpha$, interleukin- 6 and nitric oxide, preventing lipid peroxidation and reversing mitochondrial dysfunction under conditions of heightened oxidative stress. ${ }^{35}$

The cognitive effects of omega-3 fatty acids combined with a more complex lifestyle intervention, incorporating exercise, have recently been examined in the large 3-year RCT Multidomain Alzheimer Prevention Trial (MAPT). This study examined the impact of omega- 3 fatty acid supplementation $(800 \mathrm{mg} /$ day DHA $+250 \mathrm{mg} /$ day EPA) and a multidomain intervention (cognitive training, physical activity and nutrition advice and three preventative consultations), alone or in combination, compared with placebo on cognitive function in 1680 community-dwelling, non-demented adults aged $70+$ years. ${ }^{39}$ The combination of omega-3 fatty acids and the multidomain intervention enhanced cognitive performance on a secondary measure of cognitive function and in subgroup analyses of those with an increased dementia risk score, but no benefits were found for the primary cognitive endpoints. The absence of an effect on the primary outcome for the multidomain intervention may have been influenced by the design of the exercise component that was limited to generic physical activity advice to be more active (eg, aim to walk $30 \mathrm{~min}$ per day) and did not include a structured programme designed to enhance physical function.

\section{Muscle mass and strength and cognitive function}

The ageing process targets multiple systems of the body, leaving older individuals vulnerable to deterioration of brain, vascular and musculoskeletal systems. A progressive loss of muscle mass, strength and function, termed as sarcopenia, occurs with ageing. After the age of 40-50 years, there is a progressive loss of muscle mass in the magnitude of $1 \%-2 \%$ per year. ${ }^{40}$ Sarcopenia is a growing public health concern, increasing the risk of low trauma fracture, disability, morbidity and reducing quality of life in older people. ${ }^{40}$

Low muscle strength has been linked to lower global cognitive performance ${ }^{41}$ and a higher risk of cognitive impairment. ${ }^{42}$ Greater frailty has been associated with increased cognitive decline in patients with $\mathrm{AD} .{ }^{43}$ A recent investigation using data derived from the UK Biobank identified a significant relationship between grip strength and multiple cognitive domains in over 475000 individuals from the general population. ${ }^{44}$ While these findings emphasise the connection between muscular strength and brain health, more research is needed to determine whether increasing muscle mass and strength can enhance cognitive function. Results from the Study of Mental and Resistance Training (SMART) trial showed that strength improvements due to 6 months of high intensity resistance training were associated with improvements to cognitive function in older people with mild cognitive impairment. ${ }^{45}$

Combining dietary protein supplementation with resistance training exercise has been proposed as a strategy to offset muscle decline in older people. ${ }^{46}$ A 16-week RCT conducted in older women found that increasing lean red meat intake, combined with twice weekly resistance training, increased lean tissue mass and muscle strength compared with resistance training coupled with a high carbohydrate diet. $^{47} \mathrm{~A}$ recent meta-analysis has confirmed that dietary protein can augment the effects of resistance training exercise on muscle mass and strength, although the findings indicate that resistance training is the more potent stimulus for increasing muscle strength than protein intake. ${ }^{48}$ Protein dose may be an important consideration, with an RCT in men aged $>70$ years having demonstrated that providing double the recommended daily allowance (RDA) of protein $(1.6 \mathrm{~g}$ protein $/ \mathrm{kg} /$ day), compared with the RDA, for 10 weeks provided benefits to lean body mass and knee extension peak power. ${ }^{49}$ These findings are consistent with the premise that older adults need a higher intake of protein to increase muscle protein synthesis. ${ }^{46}$ With regards to the impact of combining exercise with protein on cognitive function, there is some evidence that the combination of $30 \mathrm{~g}$ daily protein supplementation and resistance training exercise for 6 months enhanced processing speed in frail and prefrail elderly. ${ }^{50}$ While a consistent benefit of protein supplementation for cognition has not been established, it has been proposed that amino acids such as tyrosine and tryptophan may be capable of modulating cognitive function. ${ }^{51}$

\section{Objective}

It is hypothesised that a multifaceted approach combining exercise with dietary modification involving omega-3 fatty acids, vitamin $\mathrm{D}$ and protein may offer cognitive benefits, relative to the control condition, as they will target complementary pathways related to neuroinflammation, neuroprotection and neuroplasticity. ${ }^{52135}$

The primary aim of this study is to investigate the efficacy of a 6-month multifaceted intervention, incorporating an exercise programme with omega-3 fatty acid, vitamin D and protein supplementation on cognitive function in older people with subjective memory complaints. The secondary aims are to: (1) determine if any beneficial effects of this intervention on cognitive function are mediated through increases in muscle strength or mass, (2) explore the impact of the intervention on other aspects of psychological and physical health and (3) evaluate the longer term residual effects (6 months after cessation of the intervention) on cognition. 


\section{METHODS/DESIGN}

\section{Study design}

This is a 12-month, community-based, parallel group, randomised, double-blind, placebo-controlled trial involving a 6-month supervised and structured intervention, followed by an additional 6-month follow-up to evaluate any long-term residual effects. A total of 148 individuals with subjective memory impairment (SMI) aged 60-85 years will be randomised to: (1) a supervised multimodal exercise programme involving progressive resistance training and aerobic exercise, combined with omega-3 fatty acid, vitamin D and protein supplementation or (2) a stretching/flexibility exercise programme and placebo supplements. During the 6-month postintervention follow-up, participants will no longer be provided with the exercise programmes or supplements but will be encouraged to continue to exercise and follow a healthy diet. This study protocol is reported in accordance with the Standard Protocol Items: Recommendations for Interventional Trials statement, ${ }^{52}$ and results will be reported in line with the Consolidated Standards of Reporting Trials (CONSORT) statement ${ }^{53}$ and the CONSORT statement for non-pharmacological interventions. ${ }^{54}$ The study was prospectively registered on the Australian New Zealand Clinical Trials Registry on 10 November 2016.

\section{Participants and screening}

Participants will be included into the study based on the following criteria: (1) presence of subjective memory complaints, defined as a positive response to the question 'do you feel like your memory is becoming worse?' and a score $>17$ on the Montreal Cognitive Assessment (MoCA), (2) community dwelling and aged 60-85 years, (3) fluent in written and spoken English and (4) with normal or corrected to normal vision and hearing.

To minimise extraneous causes of cognitive impairment, participants will be excluded based on the following: (1) diagnosis of $\mathrm{AD}$ or other dementia and Parkinson's disease; (2) history of brain damage including significant head trauma, stroke or serious neurological disorder, including loss of consciousness $>24$ hours; (3) clinical diagnosis of psychiatric disorder likely to affect cognition, including current clinical diagnosis of depression, commencement of anti-anxiety or antidepressant medication within the past 6 months, score of $>5$ on the short Geriatric Depression Scale; (4) or alcohol or drug dependency within the last 2 years. Exclusion criteria relevant to the study intervention, supplement and/or exercise programme, will include: (5) existing diagnosed gastrointestinal disorders likely to impact absorption of fatty acids (eg, coeliac disease); (6) known allergy to fish or any other component in the dietary supplement; (7) type two diabetes; (8) body mass index $>40$; (9) health conditions that contraindicate exercise or insufficient mobility to exercise; (10) participating in progressive resistance training $>1$ week or more $>150$ min per week of moderate intensity physical activity; (11) current smokers; (12) oily fish consumption ( $\geq 1 /$ week); (13) daily use of omega-3 rich supplement $>500 \mathrm{mg}$ or vitamin D supplements $>500$ IU within the past 12 weeks; (14) use of cholinesterase inhibitors, benzodiazepines, antipsychotics or high-dose antidepressants (judged by medical advisor), use of warfarin or high dose blood thinners, resistant hypertension defined as the use of more than three antihypertensive agents and medications contraindicated for use with omega-3 fatty acids, vitamin D or protein. Any uncertainty or discrepancies about participants' clinical history will be resolved by consulting with a physician familiar with the study (investigator SM).

Participants will be recruited from within the local community in Melbourne (Australia) and surrounding areas via newspaper advertisements, community presentations, social media and community notice boards. Participants will be provided with the exercise programme free of charge as an incentive to participate in the trial.

\section{Randomisation}

Participants will be randomly allocated to one of the two parallel groups using a simple randomisation computer generated random sampling set, with an allocation ratio of 1:1. Randomisation will be stratified for gender and age, separated into three categories of 60-69.9 years, 70-79.9 and $80-85.9$ years. Randomisation will be conducted by personnel not otherwise involved in the trial. Participant group allocation will be provided in an opaque envelope to the researcher involved in assigning participants to the intervention, on completion of the baseline appointment. The researcher who assigns participants to the intervention will distribute the study treatment and provide information about the assigned exercise programme. This researcher will no longer be blinded from this point.

\section{Blinding}

Both the active supplement and the placebo will be packaged in non-transparent sachets. All researchers involved in outcome assessments will be blinded to participant group allocation, with the exception of the 6-month dual-energy X-ray absorptiometry (DXA) scan, which may be conducted by an unblinded researcher due to limited qualified personnel. All DXA analyses will be carried out by a blinded researcher. During the intervention period, only unblinded researchers will be in contact with participants enrolled in the trial. In order to maintain a double-blind study design, participants will not be informed that all individuals assigned to the multimodal exercise programme will also receive the supplement drink containing omega-3, vitamin $\mathrm{D}$ and protein, nor that all participants assigned to the stretching/flexibility condition will also receive the placebo. The purpose of concealment will be explained when all participants have completed the trial. Exercise trainers delivering the exercise programme will not be informed that participants in the multimodal exercise programme are receiving the active supplements or those in the stretching group are receiving the placebo. All data analysis will be completed by personnel who are blinded to the intervention 
assignment. Participants will be unblinded after the 12-month assessment.

The codes will only be broken in an emergency, such as a severe adverse event that requires knowledge of the treatment being taken in order to manage a participant's condition. The principal investigator and ethics committee will be informed within 24 hours of the codebreak envelope being opened.

\section{Intervention}

Multimodal exercise programme plus omega-3, vitamin D and protein supplement drink

Exercise intervention: participants will take part in a 6-month group community-based programme designed by an exercise physiologist, conducted within local health and fitness centres located in Melbourne, Australia. Participants will attend $2 \times 1$ hour weekly supervised exercise sessions for 6 months at a health and fitness centre in close proximity to their home. Exercise classes will be exclusive to PONDER study participants and will not exceed 10 participants. Exercise sessions will be supervised by a dedicated, accredited exercise trainer. Each exercise session will involve a $5-10 \mathrm{~min}$ group warm up, $15 \mathrm{~min}$ of aerobic exercise followed by approximately $30-40 \mathrm{~min}$ of progressive resistance training concluding with a $5 \mathrm{~min}$ cool down. The aerobic exercises will be rotated rather than progressed. In line with current recommendations to enhance cognition, ${ }^{5}$ the progressive resistance training will consist of two sets of 8-12 repetitions, at a weight that cannot be lifted for more than these number of repetitions, at a rating of perceived exertion of 5-8 ('hard to very-hard') on the 10 -point scale..$^{55}$ This will be completed for eight resistance exercises involving dynamic concentric and eccentric contractions targeting all the major muscle groups and with an emphasis on weekly progressive overload (increments of $2 \%-10 \%$ ). Details of the multimodal programme are shown in table 1 .

Supplement: the study treatment will be a vanilla-flavoured daily supplement drink containing $5 \mathrm{~g}$ of omega-3 powder (18\% EPA, 12\% DHA equivalent to $900 \mathrm{mg}$ EPA and $600 \mathrm{mg}$ DHA), vitamin D3 $1000 \mathrm{IU}$ and $25 \mathrm{~g}$ of whey protein concentrate (WPC) 80 . The total of $1.5 \mathrm{~g}$ omega-3 fatty acids is a physiologically relevant dose (equal to half portion of farmed Atlantic salmon or a full portion of fresh or tinned wild Atlantic salmon). ${ }^{56}$ The aim of $1000 \mathrm{IU} /$ day vitamin D3 treatment is to raise serum $25(\mathrm{OH}) \mathrm{D}$ concentrations to at least $50-60 \mathrm{nmol} / \mathrm{L}$, in line with current Australian recommendations. ${ }^{57}$ When combined with resistance training, $20 \mathrm{~g}$ of whey protein isolate has been demonstrated to increase muscle protein synthesis in older people ${ }^{58}$; therefore, $25 \mathrm{~g}$ of WPC was selected to provide a comparable dose of protein. Each drink will provide approximately $590 \mathrm{~kJ}$ of energy, $2 \mathrm{~g}$ of lactose, $6 \mathrm{~g}$ of fat and $20 \mathrm{~g}$ of protein. The supplement will be provided in powder form to be mixed with $200 \mathrm{~mL}$ water. The treatments have been developed by Omniblend Australia. Participants will be instructed to

\section{Table 1 Details of the exercise programmes}

\begin{tabular}{|c|c|}
\hline & Multimodal exercise \\
\hline Frequency & $2 \times$ per week. \\
\hline Intensity & RPE rating of 5-8 (hard to very hard). \\
\hline Warm up & 5min group-based warm up including balance and mobility exercises. \\
\hline Aerobic & 15 min aerobic training rotating exercises: treadmill, bike, stepper, rower, $\mathrm{X}$-trainer/elliptical trainer and so on. \\
\hline \multirow[t]{2}{*}{ Cool down } & 5-10min cool down: relaxation type exercises incorporating stretching. \\
\hline & Stretching and flexibility. \\
\hline Warm up & 5 min light walking/marching, range of motion exercises. \\
\hline Aerobic & Nil. \\
\hline Resistance & Nil. \\
\hline Flexibility & $\begin{array}{l}\text { 35-40 min variety of light stretching/flexibility exercise and light resistance band exercises that will not } \\
\text { increase strength. }\end{array}$ \\
\hline Cool down & 5-10 min cool down: relaxation type exercises. \\
\hline
\end{tabular}

RPE, rating of perceived exertion. 
consume the supplement immediately before breakfast, except on days involving the exercise programme, when they will be requested to consume the supplement 1-2 hours after completing the session. To reduce the likelihood of any gastrointestinal side effects, participants will be instructed to consume only half the supplement dose for the first 7 days of the intervention.

\section{Control group (stretching/flexibility programme+placebo drink)}

Exercise: participants will complete a 6-month group community-based stretching/flexibility programme designed by an exercise physiologist within local health and fitness centres. Participants will attend $2 \times 1$ hour weekly supervised exercise sessions for 6 months. Groups will be exclusive to study participants. The exercise programme will involve stretching and is designed not to influence heart rate, strength or aerobic capacity but to provide a similar level of social contact and attention from a dedicated exercise trainer at each centre. Details of the stretching/flexibility programme are shown in table 1 .

Supplement: the placebo drink will be vanilla flavoured and matched for appearance and caloric content and will contain $16 \mathrm{~g}$ whole milk powder and $15 \mathrm{~g}$ maltodextrin. Each drink will provide approximately $590 \mathrm{~kJ}$ of energy.

\section{Compliance}

Compliance to the exercise programmes will be assessed using attendance records and for the multimodal group, monthly exercise cards collected from the exercise trainers. Each exercise card contains information regarding sessions attended, exercises completed, set, repetitions, time and training intensity. A compliance calendar showing each month will be provided to assess supplement compliance. Participants will mark off each day the supplement is consumed and record any reasons for variations (ie, missed supplement or taken later in the day). Participants who are non-compliant with the intervention will still be requested to attend all follow-up appointments.

\section{Adverse events}

Any adverse events potentially or confirmed to be associated, with the exercise programme will be recorded on the exercise cards provided to the exercise trainers. The supplement calendar will be used to record adverse events potentially due to the supplement. Any health-related unfavourable or unintended medical occurrence (eg, sign, symptom, syndrome and illness), deemed to be relevant to the study, that develops or worsens during the trial will be classed as an adverse event. All adverse events will be assessed for seriousness, severity, causality and expected outcome by the researchers. There will be no formal data monitoring committee, due to the size of the study and the single site; however, all data will be reviewed by the research team at regular intervals throughout the study.

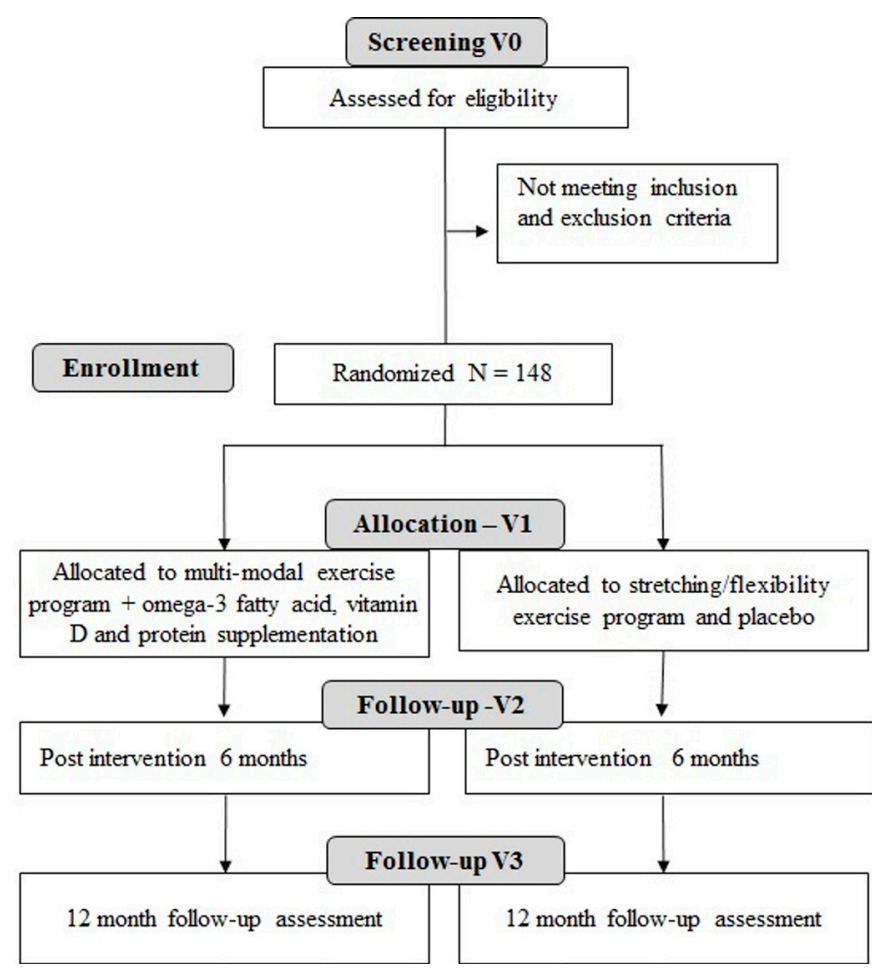

Figure 1 Flow diagram: participant recruitment and screening flow chart.

\section{Sample size calculation}

To date, no studies have investigated the cognitive effects of the specific intervention combination examined in the current study. For this reason, the anticipated effect size is largely based on findings derived from the exercise literature. Exercise exerts an average 0.5 SD effect size (equating to a medium effect size) on executive function. ${ }^{5}$ A recent meta-analysis has indicated that multimodal exercise interventions of at least 4 weeks' duration have a positive effect on cognition in the order of a 0.33 effect size, in people over the age of 50 years. ${ }^{10}$ Based on these figures, we anticipate at least a medium effect size $(\mathrm{f}=0.25)$. Allowing for $15 \%$ attrition rate, a sample of 148 participants, with 74 individuals assigned to each study arm, will provide a power level of 0.8 at the $5 \%$ probability level.

\section{Procedure}

Participants will attend a total of four visits to Deakin University in Melbourne to undergo study assessments (see figure 1). All participants will be requested to abstain from alcohol for 24 hours prior to study visits. No caffeine is to be consumed 2 hours prior to study visits, and participants will consume the same food and drink at the baseline (V1) and 6-month (V2) visits. Baseline and 6-month visits will be held at the approximate same time of day (within 2hours).

- Prescreening: all participants will undergo telephone screening.

- Screening (V0): potential participants who meet the prescreening study criteria will attend a face-to-face session with a researcher to provide written informed 
consent and undergo screening for inclusion/exclusion criteria. Eligible individuals will undergo familiarisation of core study measures and will be provided with a physical activity monitor and pathology collection requests to complete blood collection prior to V1. Participants requiring medical approval must obtain this prior to baseline.

- Baseline (V1): to be conducted within 4 weeks of the V0 screening visit. During V1, cognitive assessments will be carried out first, followed by cardiovascular measures, anthropometry, DXA, functional measures and questionnaires. On completion of V1, participants will be allocated to either the intervention group or control group and provided with the supplement and details of the relevant gym programme. Participants are requested not to commence additional omega-3 or vitamin supplementation during the trial or to take part in more than one additional structured exercise class per week.

- Intervention period: an unblinded study member will remain in contact with participants via telephone and face-to-face visits at the gyms and will collect exercise cards, including adverse event logs and supplement compliance logs. Possible adverse events, gym non-attendance and supplement non-compliance will be followed up by telephone by an unblinded researcher.

- 6 month follow-up (V2): participants will repeat physical activity monitoring and blood collection within the week prior to V2. All measures from baseline will be repeated along with the MaCA.

- 12-month follow-up (V3): due to funding constraints, only cognitive assessments including the Cogstate, Trail Making Test and MoCA and study questionnaires will be completed at V3.

\section{Primary outcome}

Change in global cognitive function derived from the CogState computerised battery and the Trail Making Test after 6 months.

\section{Secondary outcomes}

Changes in cognitive function for the six individual measures and the composite scores of working memory/ learning and attention/psychomotor function assessed using the CogState battery, the Trail Making Test, the MoCA after 6 months and 12 months, and changes in mood, health-related quality of life, strength, physical function, body composition, cardiovascular health and sleep quality after 6 months. A list of outcomes and time points are shown in table 2 .

\section{Screening measures}

The self-administered 15-item Short Form Geriatric Depression Scale (GDS) ${ }^{59}$ will be used to screen for the presence of depression at V0. Scores greater than 5 potentially indicate depression and those who score $>5$ will not be eligible for the study. The interviewer administered $\mathrm{MoCA}^{60}$ will be used to screen for the possible presence of dementia. The MoCA is scored out of 30 and assesses short-term memory, visuospatial ability, executive function, verbal fluency, attention and working memory and orientation to time and place and is summed to create a global measure of cognition. Participants require a score $>17$ to be eligible for the study. The MoCA has been shown to have $90 \%$ sensitivity and $87 \%$ specificity for detecting MCI $(\mathrm{n}=94)$ and $100 \%$ and $87 \%$, respectively, for detecting early $\mathrm{AD} .^{60}$ The MOCA will be used for screening purposes at $\mathrm{V} 0$ and repeated as a secondary outcome after 6 months (V2) and 12 months (V3).

\section{Primary outcome measures \\ Cognitive assessments}

The Cogstate Computerised Battery (www.cogstate. com) will be used to assess cognition for six cognitive tasks, which will form the basis for two composite scores (working memory/learning and attention/psychomotor function) and a measure of global cognitive function, which will be the primary outcome. The individual cognitive tasks include the Detection Task (reaction time task measuring psychomotor function), the Identification Task (choice reaction time task measuring visual attention), the One Card Learning Task (visual recognition memory and attention) and the One-Back Task (working memory and attention). For all tasks, speed (reaction time in milliseconds) and accuracy (number of correct responses made) will be recorded. The fifth task is the Groton Maze Task, which assesses executive function and is calculated based on the number of errors. The final task is the International Shopping List (verbal learning and memory), which is scored using accuracy, calculated by summing correct responses. Individual raw scores for each task will be transformed into a z-score using the mean and SD of the total sample in the study. Composite scores for working memory/learning will be calculated by averaging the standardised scores (z-score) for the One Card Learning and One-Back Tasks, and the attention/ psychomotor function composite will be calculated by averaging the z-scores for the Detection and Identification Tasks. Global cognitive function will be derived as the average z-scores from all six tests, which higher scores indicating better performance. The CogState computerised tasks are specifically designed for repeated administration over short time periods and have been validated in older adults. ${ }^{61-63}$ Practice or fatigue effects are minimal for these tasks, making them suitable for detecting subtle cognitive changes. ${ }^{61-63}$ To further minimise practice effects, the entire battery will be practised at V0.

The Trail Making Test ${ }^{64} 65$ Part A (visuoperceptual ability) involves drawing lines to connect numbered dots in ascending numerical order as quickly as possible while still maintaining accuracy. Part B (working memory and task-switching ability) involves connecting dots in ascending order, alternating between numbers and letters as quickly as possible. The score is the number of seconds taken to complete each part of the test. The score from Part B minus Part A provides an indication of executive 
Table 2 Outcome measures and relevant data collection time points

\begin{tabular}{|c|c|c|c|c|c|}
\hline Outcome measures & Data collection method & $\begin{array}{l}\text { Visit 0: } \\
\text { practice }\end{array}$ & $\begin{array}{l}\text { Visit 1: } \\
\text { baseline }\end{array}$ & $\begin{array}{l}\text { Visit 2: } \\
\text { 6month }\end{array}$ & $\begin{array}{l}\text { Visit 3: } \\
\text { 12month }\end{array}$ \\
\hline \multicolumn{6}{|l|}{ Primary outcome } \\
\hline \multirow[t]{2}{*}{ Cognitive function } & Cogstate Computerised Battery & Practice & $\mathrm{X}$ & $x$ & $\mathrm{x}$ \\
\hline & Trail Making Test & Practice & $\mathrm{X}$ & $x$ & $x$ \\
\hline \multirow{3}{*}{$\begin{array}{l}\text { Additional outcomes } \\
\text { Cognitive function }\end{array}$} & Montreal Cognitive Assessment & $x$ & & $x$ & $x$ \\
\hline & NART-R IQ & $x$ & & & \\
\hline & Cognitive Function Instrument & & $x$ & $x$ & $x$ \\
\hline \multirow[t]{2}{*}{ Muscle strength } & 3RM Knee extension & Practice & $x$ & $x$ & \\
\hline & Hand grip strength & & $x$ & $x$ & \\
\hline \multirow[t]{4}{*}{ Physical function } & Four Metre Walk Test & & $x$ & $x$ & \\
\hline & Four-Square Step Test & & $\mathrm{X}$ & $x$ & \\
\hline & 30 s Sit-to-Stand Test & & $x$ & $x$ & \\
\hline & Timed Up and Go Test & & $x$ & $x$ & \\
\hline \multirow[t]{2}{*}{ Mood } & Short Geriatric Depression Scale & $x$ & & & \\
\hline & Depression Anxiety Stress Scale & & $\mathrm{X}$ & $x$ & $x$ \\
\hline Quality of life & Short-form (SF)-36 version 2 & & $x$ & $x$ & $\mathrm{x}$ \\
\hline Sleep & Pittsburg Sleep Quality Index & & $x$ & $x$ & $\mathrm{x}$ \\
\hline Personality & Short NEO-Personality Inventory & $x$ & & & \\
\hline Demographics/lifestyle & Lifestyle Questionnaire & & $\mathrm{x}$ & $x$ & $\mathrm{x}$ \\
\hline \multirow[t]{2}{*}{ Anthropometry } & Body mass index & & $x$ & $x$ & \\
\hline & Hip, waist and neck circumference & & $x$ & $x$ & \\
\hline Body composition & DXA (total body and regional) & & $x$ & $x$ & \\
\hline \multirow[t]{2}{*}{ Cardiovascular function } & Resting blood pressure & & & & \\
\hline & $\begin{array}{l}\text { Central arterial pressure, pulse wave velocity and } \\
\text { arterial stiffness }\end{array}$ & & $x$ & $x$ & \\
\hline \multirow[t]{2}{*}{ Diet } & ASA-24 Dietary Assessment Tool & & $x$ & $\mathrm{x}$ & $x$ \\
\hline & PUFA Questionnaire & & $x$ & $x$ & \\
\hline \multirow[t]{2}{*}{$\begin{array}{l}\text { Habitual } \\
\text { physical activity }\end{array}$} & CHAMPS Survey & & $x$ & $x$ & $\mathrm{x}$ \\
\hline & 7-day physical activity (Actigraph) & & $x$ & $x$ & $x$ \\
\hline \multirow[t]{3}{*}{ Biomarkers } & Cholesterol and HsCRP & & $x$ & $x$ & \\
\hline & Omega- 3 fatty acids and vitamin $\mathrm{D}$ & & $x$ & $x$ & \\
\hline & Apolipoprotein E e4 status & & $x$ & & \\
\hline
\end{tabular}

3RM, three repetition maximum; ASA-24, Automated Self-Administered 24 hours; CHAMPS, Community Healthy Activities Model Program for Seniors; DXA, dual-energy X-ray absorptiometry; HsCRP, high-sensitity C reactive protein; NART-R IQ, National Adult Reading Test Revised IQ; PUFA, polyunsaturated fatty acids.

function ${ }^{65}$ and will be considered the primary outcome generated from the Trail Making test. The Trail Making Test has test-retest reliability ranging from 0.74 to $0.85 .{ }^{66}$ To minimise practise effects, the Trail Making Test will be practised at V0.

\section{Secondary outcome measures}

Mood, quality of life and sleep

The 21-item Depression, Anxiety and Stress Scale (DASS $)^{67}$ will be used to assess mood. The DASS consists of three subfactors that yield subscores for each domain: depression, anxiety and stress. Higher total scores indicate a higher degree of mood dysfunction.

The self-administered Short Form 36 version 2 (SF-36 v2) questionnaire will be used to measure health-related quality of life. ${ }^{68}$ The SF-36 covers the domains of physical functioning, role-physical, bodily pain, general health, vitality, social functioning, role-emotional and mental health. The current study will report Australian normbased scores using previously published guidelines ${ }^{69}$ to provide each domain score a standardised mean of 50 
and a SD of 10 . Separate quality of life summary scores will be calculated for the physical component and the mental component.

The self-administered 19-item Pittsburgh Sleep Quality Index (PSQI) will be used to measure sleep quality and disturbance over a 4 -week period. ${ }^{70}$ The scale is composed of items that generate seven subscores: subjective sleep quality, sleep latency, sleep duration, habitual sleep efficiency, sleep disturbance, use of sleep medications and daytime dysfunction. Both the total score and subscores will be outcomes for this measure.

\section{Muscle strength}

Lower body muscle strength will be determined by the Keiser bilateral knee extension machine (Keiser Corporation, Fresno, California, USA), using a standard three repetition maximum protocol (see ref 71). Participants will warm up with 8 repetitions at $3 \mathrm{~kg}$. The weight will be increased progressively to participant's estimated three repetition maximum $(\mathrm{kg})$. After a 2 min rest, resistance will be increased (or decreased) until only three repetitions in good form can be completed.

Bilateral maximal isometric grip strength will be measured using a hand-held dynamometer (Jamar dynamometer, Asimov Engineering Co, Los Angeles, California, USA). In a seated position, with elbow flexed at $90^{\circ}$, forearm neutral and hand slightly extended, participants will be instructed to squeeze the hand grip as tightly as possible and release for three trials with a 1 min rest between trials. After a practise trial, the maximal grip strength $(\mathrm{kg})$ of each hand will be recorded. The test is performed by both the dominant and non-dominant limbs.

\section{Physical function}

All functional tests will be preceded by a practise trial. The $4 \mathrm{~m}$ walk test will be used to assess gait speed. Participants walk in a straight line at their normal walking speed across four metres with speed recorded using timing gates (Swift Speedlink Performance Equipment systems). The outcome will be the fastest time measured in seconds (to the nearest millisecond) from two trials.

The Four-Square Step Test (FSST) will be used to assess dynamic standing balance and stepping speed in four different directions. ${ }^{72}$ The FSST is completed first in a clockwise and then counter-clockwise direction. Participants will complete two test trials, and the outcome will be the fastest time (in seconds) taken to complete the sequence.

The Timed Up and Go Test measures dynamic balance during three commonly performed functional activities: standing up from and sitting down in a chair, walking and turning. ${ }^{73}$ Participants are required to stand up from a chair, walk as quickly as possible around a cone (marking $3 \mathrm{~m}$ ), turn and walk back to the chair and sit down. The outcome will be the time (in seconds) to complete the sequence.
The $30 \mathrm{~s}$ Sit-to-Stand Test $^{74}$ will be used to assess lower extremity muscle strength and power. Starting from a seated position in a chair, chair measuring $45 \mathrm{~cm}$ from the ground, with arms folded across the chest, participants stand fully upright and then return to the seated position as many times as possible in $30 \mathrm{~s}$. The outcome is the number of sit-to-stands completed in $30 \mathrm{~s}$.

\section{Anthropometrics and body composition}

Height will be measured in centimetres using a stadiometer fixed to the wall, and weight will be measured in kilograms using calibrated scales with standardised protocols. Body mass index (BMI) will be calculated as weight $(\mathrm{kg}) /$ height $\left(\mathrm{m}^{2}\right)$. Waist and hip circumference will be measured in centimetres using the WHO protocol. ${ }^{75}$ Waist-to-hip ratio will be calculated by dividing the waist circumference measurement by the hip circumference. Neck circumference will be measured around the midpoint of the participant's neck, between the midcervical spine and mid anterior neck. All measures will be taken twice and the average value used as the outcome.

Dual-energy X-ray Absorptiometry (DXA) will be used to assess body composition (fat, lean and bone) (Lunar iDXA, version Encore 16.2, GE Healthcare Medicine, Wisconsin, USA). Total body lean mass, fat mass and percentage body fat will be determined from the total a regional (arm and legs) body. Standardised procedures (2015 ISCD official positions) for participant positioning and scan analysis will be used for all scans (https://www. iscd.org/official-positions/2015-iscd-official-positionsadult/). During each visit participants will be scanned in a hospital gown and instructed to remove all metal objects. All scans for all participants will be performed by the same operator on the same scanner following daily quality assurance and control monitoring.

\section{Cardiovascular health}

Seated blood pressure will be assessed using an ambulatory blood pressure monitor. The blood pressure cuff will be fixed to the left arm following a 5 min period of seated rest. Four consecutive measurements will be taken at $1 \mathrm{~min}$ intervals and the average of the final three readings will be used as the outcome.

The SphygmoCor XCEL (AtCor Medical PTY) will be used to measure central arterial pressure waveform, pulse wave velocity (PWV) and arterial stiffness. Pulse wave analysis (PWA) will be measured after 5 min of quiet supine rest with the cuff attached to the left arm held in a straight position. Measures will be taken twice and the average value used at the outcome. To conduct carotid-femoral PWV, a cuff will be placed around the femoral artery to capture the femoral waveform, and a tonometer will capture the carotid waveform. The distance between the carotid and femoral arteries will be measured, and the velocity automatically calculated by dividing the distance by the pulse transit time. Measures will be taken twice, with a third measure completed if the difference between 
the $\mathrm{PWV}$ readings is $>0.5 \mathrm{~m} / \mathrm{s}$. The average value will be used at the outcome.

\section{Demographic variables and potential covariates}

Demographic and lifestyle information will be collected on participant's ethnic and educational background, employment history/status, medical history, history of falls and fractures, family history of osteoporosis, current medication and dietary supplement use, alcohol intake, weekly television viewing and sitting time and sun exposure habits.

\section{Cognitive and psychological factors}

The National Adult Reading Test-Revised (NART-R) will be used to estimate IQ ${ }^{76}$ The NART-R is suitable to assess premorbid intellectual functioning in the presence of cognitive impairment. ${ }^{77}$ The number of errors in pronunciation will be converted to an estimate of FullScale IQ score. The Cognitive Function Instrument ${ }^{78}$ is a self-report measure of 14 items that probe experiences of memory decline over the past year. This measure will be used to provide greater detail regarding subjective cognitive complaints and has been demonstrated to correlate with objective cognitive decline.$^{79}$ The short 60 -item NEO Personality Inventory will be used to assess the five major personality domains of extraversion, agreeableness, conscientiousness, neuroticism and openness to experience $^{80}$ as personality characteristics may contribute to subjective cognitive complaints. ${ }^{81}$

\section{Blood biomarkers}

Within 7 days prior to baseline (V1) and 6 months (V2), participants will attend a commercial pathology clinic collection centre where a fasted, morning (08:00-10:00) venous blood sample will be collected. Blood samples will be separated into serum/plasma and red blood cells by low speed centrifugation $\left(3000 \mathrm{rpm}, 4^{\circ} \mathrm{C}\right.$ for $10 \mathrm{~min}$ ). The following samples will be sent to a central pathology laboratory accredited by the National Association of Testing Authorities Royal College of Pathologists Australasia: serum total cholesterol, high-density lipoprotein (HDL)-cholesterol and triglycerides will be analysed using an enzymatic colorimetric method (Roche Diagnostics, Mannheim). Low-density lipoprotein (LDL)-cholesterol will be calculated using Friedewald's formula. Serum creatinine, urea, albumin, calcium, phosphorus, sodium, potassium and phosphorus will be analysed using standardised techniques. Additional packed red blood cells, plasma and serum samples will be stored until analysed. Samples will be stored in a $-70^{\circ} \mathrm{C}$ freezer prior to being transferred to Deakin University, where samples will be stored at $-80^{\circ} \mathrm{C}$ until the study is complete. Erythrocyte levels of omega-3 PUFAs will be extracted based on LePage and Roy method. ${ }^{82}$ The extracted fatty acids will be separated by gas chromatography, identified by the comparison with known fatty acid standards and quantified by comparison to the internal standard (21:0). Serum
$25(\mathrm{OH}) \mathrm{D}$ will be used to determine changes in vitamin $\mathrm{D}$ levels due to the supplement.

\section{Apolipoprotein E (ApoE) status}

ApoE is involved in lipid homeostasis, particularly in the determination of the levels of LDL and HDL cholesterol, which are directly and inversely correlated with risk for cardiovascular disease respectively. ${ }^{83}$ Carriers of the ApoE e4 allele have increased coronary risk, ${ }^{84}$ and e4 carriers also have a higher risk of developing late onset $\mathrm{AD}{ }^{85}$ Although e4 carriers may experience a similar benefit from lifestyle based interventions, their rate of cognitive change may be greater than in non-carriers. ${ }^{86}$ ApoE status will be assessed at baseline (V1), DNA will be extracted from venous whole blood samples using commercially available kits.

\section{Physical activity}

The Community Healthy Activities Model Program for Seniors (CHAMPS) physical activity questionnaire will be used to calculate total leisure and recreational physical activity time. This measure has been designed for use in older adults and is reliable, valid and sensitive to change. ${ }^{87}$ Individuals will document their weekly frequency and duration of participation in a typical week' of the preceding 4 weeks. Outcomes will be hours per week, and estimated kilojoules per week, spent in moderate to high intensity activities.

To obtain an objective measure of physical activity, participants will be provided with an Actigraph monitor to wear for a period of up to 7 days on three occasions. The Actigraph is a small lightweight monitor that will be worn on the right hip to measure quantity and intensity of physical activity. Monitors will be removed for sleep. Participants will complete a log of their activity including sleep hours to aid in interpretation of this data. Physical activity information will be collected for the 7 days prior to V1, V2 and V3 assessments. Time spent in sedentary, light, moderate and vigorous activity will be calculated.

\section{Diet}

The Automated Self-Administered 24hours (ASA24) Australia Dietary Assessment Tool (https://epi.grants. cancer.gov/asa24/) developed by the National Cancer Institute will be used as a 24-hour dietary recall. The ASA24 is completed online; however, it will be conducted over the phone with a researcher if participants do not have computer and internet access. The ASA24 allows respondents to report foods and drinks and amounts consumed by browsing a food category or searching from a list of food and drink terms derived from the 2007-2008 National Health and Nutrition Examination Survey. The ASA-24 provides a breakdown of macronutrients and energy, vitamins, minerals, carotenoids, fats, cholesterol and fatty acids.

A validated online questionnaire will be used to assess dietary PUFA intake. The PUFA questionnaire is a simple non-invasive method that estimates the intakes of omega-3 
and omega-6 PUFAs in a healthy adult population. ${ }^{88}$ The questionnaire includes main food sources of $\alpha$-linolenic acid as well as omega-6 PUFA, linoleic acid (LA) and arachidonic acid (AA). The PUFA questionnaire consists of 38 questions relating to the usual dietary habits related to PUFA intake over 3 months. The questions pertain to foods, such as fish, meat and eggs for omega-3 PUFA, and vegetable oils and fats for omega-6 PUFA. The questionnaire covers fish oil supplement consumption and products with added omega-3 such as bread, eggs and milk.

\section{Statistical analysis}

Data will be analysed using SPSS V.25 and STATA V.15 applying standard statistical thresholds $(\mathrm{p}<0.05)$, corrected for multiple comparisons where appropriate. There will be no interim data analysis. All data will be checked for normality prior to analysis. Data will be analysed based on an intention-to-treat approach using linear mixed models with random effects. Changes in the primary outcomes (CogState global cognitive function and Trail making test of executive function), and all secondary outcomes will be expressed as the absolute or percentage difference for the change between the intervention group and control group after 6 months or 12 months, as appropriate. For the cognitive outcomes, the results will be analysed adjusted for age, gender and years of education (model 1) as well as baseline MoCA score and ApoE status (model 2). Per-protocol analysis will be used to determine the effectiveness of the intervention among participants who are considered to be compliant with the intervention. Participants who attended $67 \%$ (two-thirds) of exercise sessions and consumed $80 \%$ of the allocated supplement will be classified as adherent for the purpose of completer analysis. All data will be presented as mean $\pm \mathrm{SD}$ or $95 \%$ CIs.

\section{Ethical approval, data management and dissemination plan}

The current protocol is based on version 4, April 2017. The first participants were enrolled into the study on 4 April 2017. In line with the ethical approval for this study, all data will be de-identified and stored in a locked filing cabinet or on a secure data drive at Deakin University. Only members of the research team will have access to the data. Only study investigators will have access to the final data set. Any changes to the trial protocol will be reported to Deakin University Human Research Ethics Committee and updated on the clinical trial registry. On completion of the trial, participants will receive a report outlining major study findings. They may also request pathology results. Study investigators will disseminate group results to the public in the form of journal articles, conference presentations, seminars and community presentations.

\section{Patient and public involvement}

Participants were not explicitly involved in the development of the trial. Feedback will be sought from all participants at the end of the study to assess burden of intervention and to help develop future trials. Participants will receive individual result summaries of key measures and a summary of the overall study findings.

\section{DISCUSSION}

Given that dementia is among one of the major causes of disability and death worldwide, ${ }^{89}$ interventions that prevent cognitive decline and delay the onset of dementia and related disorders are urgently needed. This study will be the first to evaluate whether a multifaceted intervention comprising of exercise combined with omega-3, vitamin $\mathrm{D}$ and protein supplementation can improve cognitive function in older adults at risk of cognitive decline and provide information about potential underlying mechanisms. This study will evaluate the longer-term cognitive impact of the multifaceted intervention.

Age-related impairments to cognitive and physical function are multifactorial; therefore, multidomain interventions that target multiple risk factors are needed to address a wider range of mechanisms involved in cognitive and functional decline. Promising results have been obtained from the large-scale, 2-year Finnish Geriatric Intervention Study to Prevent Cognitive Impairment and Disability (FINGER) trial, which examined the impact of a multidomain intervention on cognitive function in 1260 older adults aged 60-77 years, selected on the basis of elevated risk for dementia. ${ }^{90}$ The study intervention included four components including personalised and group dietary recommendations, an individually tailored exercise programme targeting progressive muscle strength and aerobic exercise, group and web-based cognitive training and management of metabolic and vascular risk factors compared with regular health advice. The results of this study demonstrated a significant benefit of the multidomain intervention to a composite cognitive score, a processing speed and executive function composite score, with improved or maintained cognitive function from $25 \%$ to $150 \%$ better than the control group. The only cognitive domain not to demonstrate an improvement was memory. These findings are important as they suggest that making multiple lifestyle modifications can, in turn, modify cognition.

While the results of the MAPT did not show benefits of a combined multidomain and omega-3 fatty acid intervention to the primary cognitive outcomes, some cognitive improvements were seen for secondary outcomes and subgroup analyses. Currently, the SYNchronizing Exercises, Remedies in Gait and Cognition (SYNERGIC) trial is underway to investigate whether a 20-week multimodal intervention combining aerobic and resistance training exercise, with or without cognitive training or vitamin D supplementation, can improve cognition and reduce falls in 200 adults with mild cognitive impairment. ${ }^{91}$ Similar to the current study, the SYNERGIC trial is focused on interventions that simultaneously target cognitive health and physical function. A common element of these studies is that they have incorporated cognitive training in the 
multidomain interventions. Yet the addition of cognitive training to resistance training was less effective than exercise alone for cognitive function in the SMART trial. ${ }^{13}$ The premise of 'overdosing' by including too many lifestyle intervention components has been suggested as a factor that may have contributed to these findings. ${ }^{92}$ In the current study, we have opted to limit the intervention to exercise and dietary supplementation and not include cognitive training. The dietary component of the PONDER study is also restricted to a supplement, rather than individualised dietary counselling in order to simplify the lifestyle changes required to be undertaken by participants.

Similar to the FINGER study, ${ }^{90}$ a potential limitation of the current study is that it was not feasible to examine the isolated effects of the exercise programme and dietary supplement in separate study arms. However, the study aim is to evaluate the combined impact of the exercise programme and dietary supplement on cognitive function. The duration of the PONDER intervention is shorter than the larger FINGER and MAPT trials; however, the SMART trial demonstrated cognitive benefits after only 6 months of resistance training ${ }^{13}$ indicating our study intervention duration should be sufficient to modify cognitive function. Furthermore, our study includes an additional 12-month follow-up to capture residual effects of the intervention. Finally, our study criteria does not exclude individuals with mild cognitive impairment, only those with dementia thus may include participants in the early stages of cognitive decline or preclinical dementia.

To summarise, this is the first RCT to investigate the combined effects of a multimodal exercise programme, omega-3 fatty acid, vitamin D and protein supplementation on cognitive function in older people with SMI. This study will contribute to a growing research field which assesses the effectiveness of multidomain, lifestyle-based, interventions for cognitive decline in the elderly.

\section{Author affiliations}

${ }^{1}$ Institute for Physical Activity and Nutrition (IPAN), School of Exercise and Nutrition Sciences, Deakin University, Geelong, Victoria, Australia

${ }^{2}$ Australian Institute for Musculoskeletal Science (AIMSS), The University of

Melbourne and Western Health, St Albans, Victoria, UK

${ }^{3}$ Faculty of Science Medicine and Health, University of Wollongong, Wollongong, New South Wales, Australia

${ }^{4}$ Monash Cardiovascular Research Centre, Monash HEART, Monash University, Melbourne, Australia

Contributors All listed authors fulfil the ICMJE guidelines for authorship made substantial contributions to conception and design of the trial. The exercise programme was developed by SB and RLD. SM is the medical overseer of the trial. HM drafted the article, and all authors revised the manuscript critically for important intellectual content and provided final approval of the version to be published.

Funding This work was supported by National Health and Medical Research Council - Australian Research Council (NHMRC-ARC) grant number (APP1097696). In kind support was provided by Omniblend Australia for supplements. The funding body has had no role in study design; data collection, management and will have no role in analysis, and interpretation of data; writing of the report; and the decision to submit the report for publication.

Competing interests None declared.

Patient consent for publication Not required.
Ethics approval Ethical approval was obtained from the Deakin University Human Research Ethics Committee.

Provenance and peer review Not commissioned; externally peer reviewed.

Open access This is an open access article distributed in accordance with the Creative Commons Attribution Non Commercial (CC BY-NC 4.0) license, which permits others to distribute, remix, adapt, build upon this work non-commercially, and license their derivative works on different terms, provided the original work is properly cited, appropriate credit is given, any changes made indicated, and the use is non-commercial. See: http://creativecommons.org/licenses/by-nc/4.0/.

\section{REFERENCES}

1. Prince $\mathrm{M}$, Bryce R, Albanese $\mathrm{E}$, et al. The global prevalence of dementia: a systematic review and metaanalysis. Alzheimers Dement 2013;9:63-75.

2. AlHW, Australia's health 2013. The thirteenth biennial health report of the Australian Institute of Health and Welfare (AlHW). Canberra, 2012.

3. Livingston G, Sommerlad A, Orgeta V, et al. Dementia prevention, intervention, and care. The Lancet 2017;390:2673-734.

4. Jack CR, Knopman DS, Jagust WJ, et al. Hypothetical model of dynamic biomarkers of the Alzheimer's pathological cascade. Lancet Neurol 2010;9:119-28.

5. Chang YK, Pan CY, Chen FT, et al. Effect of resistance-exercise training on cognitive function in healthy older adults: a review. $J$ Aging Phys Act 2012;20:497-517.

6. Kennedy G, Hardman RJ, Macpherson H, et al. How Does Exercise Reduce the Rate of Age-Associated Cognitive Decline? A Review of Potential Mechanisms. J Alzheimers Dis 2017;55:1-18.

7. Sofi F, Valecchi D, Bacci D, et al. Physical activity and risk of cognitive decline: a meta-analysis of prospective studies. J Intern Med 2011;269:107-17.

8. de Bruijn RF, Schrijvers EM, de Groot KA, et al. The association between physical activity and dementia in an elderly population: the Rotterdam Study. Eur J Epidemiol 2013;28:277-83.

9. Chang YK, Labban JD, Gapin JI, et al. The effects of acute exercise on cognitive performance: a meta-analysis. Brain Res 2012;1453:87-101.

10. Northey JM, Cherbuin N, Pumpa KL, et al. Exercise interventions for cognitive function in adults older than 50: a systematic review with meta-analysis. Br J Sports Med 2018;52:154-60.

11. Panza GA, Taylor BA, MacDonald HV, et al. Can Exercise Improve Cognitive Symptoms of Alzheimer's Disease? J Am Geriatr Soc 2018;66:487-95.

12. Barha CK, Galea LA, Nagamatsu LS, et al. Personalising exercise recommendations for brain health: considerations and future directions. Br J Sports Med 2017;51:636-9.

13. Fiatarone Singh MA, Fiatarone Singh MA, Gates N, et al. The Study of Mental and Resistance Training (SMART) Study-Resistance Training and/or Cognitive Training in Mild Cognitive Impairment. JAMDA 2014;15:873-80.

14. Nagamatsu LS, Chan A, Davis JC, et al. Physical activity improves verbal and spatial memory in older adults with probable mild cognitive impairment: a 6-month randomized controlled trial. J Aging Res 2013;2013:1-10.

15. Vaughan $\mathrm{S}$, Wallis M, Polit D, et al. The effects of multimodal exercise on cognitive and physical functioning and brain-derived neurotrophic factor in older women: a randomised controlled trial. Age Ageing 2014;43:623-9.

16. Kelly ME, Loughrey D, Lawlor BA, et al. The impact of exercise on the cognitive functioning of healthy older adults: a systematic review and meta-analysis. Ageing Res Rev 2014:16:12-31.

17. Wu S, Ding Y, Wu F, et al. Omega-3 fatty acids intake and risks of dementia and Alzheimer's disease: a meta-analysis. Neurosci Biobehav Rev 2015;48:1-9.

18. Goodwill AM, Szoeke C. A Systematic Review and Meta-Analysis of The Effect of Low Vitamin D on Cognition. J Am Geriatr Soc 2017;65:2161-8

19. Jayedi A, Rashidy-Pour A, Shab-Bidar S. Vitamin d status and risk of dementia and alzheimer's disease: A meta-analysis of doseresponse. Nutr Neurosci 2018;11:1-10.

20. Patrick RP, Ames BN. Vitamin D and the omega-3 fatty acids control serotonin synthesis and action, part 2: relevance for ADHD, bipolar disorder, schizophrenia, and impulsive behavior. Faseb $J$ 2015;29:2207-22.

21. Bazinet RP, Layé S. Polyunsaturated fatty acids and their metabolites in brain function and disease. Nat Rev Neurosci 2014;15:771-85.

22. Simopoulos AP. Omega-3 fatty acids in health and disease and in growth and development. Am J Clin Nutr 1991;54:438-63. 
23. Meyer BJ. Australians are not meeting the recommended intakes for omega-3 long chain polyunsaturated fatty acids: Results of an analysis from the 2011-2012 national nutrition and physical activity survey. Nutrients 2016;8:111.

24. Sheppard KW, Cheatham CL. Omega- 6 to omega-3 fatty acid ratio and higher-order cognitive functions in 7- to 9-y-olds: a crosssectional study. Am J Clin Nutr 2013;98:659-67.

25. Andruchow ND, Konishi K, Shatenstein B, et al. A lower ratio of omega- 6 to omega- 3 fatty acids predicts better hippocampusdependent spatial memory and cognitive status in older adults. Neuropsychology 2017;31:724-34.

26. Fotuhi M, Mohassel P, Yaffe K. Fish consumption, long-chain omega-3 fatty acids and risk of cognitive decline or Alzheimer disease: a complex association. Nat Rev Neurol 2009;5:140-52.

27. Yurko-Mauro K, McCarthy D, Rom D, et al. Beneficial effects of docosahexaenoic acid on cognition in age-related cognitive decline. Alzheimers Dement 2010;6:456-64.

28. Sinn N, Milte CM, Street SJ, et al. Effects of $n-3$ fatty acids, EPA v. $\mathrm{DHA}$, on depressive symptoms, quality of life, memory and executive function in older adults with mild cognitive impairment: a 6-month randomised controlled trial. Br J Nutr 2012;107:1682-93.

29. Stonehouse W. Does Consumption of LC Omega-3 PUFA Enhance Cognitive Performance in Healthy School-Aged Children and throughout Adulthood? Evidence from Clinical Trials. Nutrients 2014:6:2730-58.

30. Dangour AD, Allen E, Elbourne D, et al. Effect of 2-y n-3 long-chain polyunsaturated fatty acid supplementation on cognitive function in older people: a randomized, double-blind, controlled trial. Am J Clin Nutr 2010;91:1725-32

31. Danthiir V, Hosking DE, Nettelbeck T, et al. An 18-mo randomized, double-blind, placebo-controlled trial of DHA-rich fish oil to prevent age-related cognitive decline in cognitively normal older adults. Am J Clin Nutr 2018;107:754-62.

32. Afzal S, Bojesen SE, Nordestgaard BG. Reduced 25-hydroxyvitamin $D$ and risk of Alzheimer's disease and vascular dementia. Alzheimers Dement 2014;10:296-302.

33. Annweiler C, Rolland Y, Schott AM, et al. Serum vitamin D deficiency as a predictor of incident non-Alzheimer dementias: a 7-year longitudinal study. Dement Geriatr Cogn Disord 2011;32:273-8.

34. Annweiler C, Schott AM, Rolland Y, et al. Dietary intake of vitamin $\mathrm{D}$ and cognition in older women: a large population-based study. Neurology 2010;75:1810-6.

35. Anastasiou CA, Yannakoulia M, Scarmeas N. Vitamin D and cognition: an update of the current evidence. J Alzheimers Dis 2014;42 Suppl 3:S71-S80.

36. Pettersen JA. Does high dose vitamin D supplementation enhance cognition?: A randomized trial in healthy adults. Exp Gerontol 2017;90:90-7.

37. Annweiler $\mathrm{C}$, Beauchet $\mathrm{O}$. Vitamin d in older adults: the need to specify standard values with respect to cognition. Front Aging Neurosci 2014;6.

38. Wu A, Ying Z, Gomez-Pinilla F. Docosahexaenoic acid dietary supplementation enhances the effects of exercise on synaptic plasticity and cognition. Neuroscience 2008;155:751-9.

39. Andrieu S, Guyonnet S, Coley N, et al. Effect of long-term omega 3 polyunsaturated fatty acid supplementation with or without multidomain intervention on cognitive function in elderly adults with memory complaints (MAPT): a randomised, placebo-controlled trial. Lancet Neurol 2017;16:377-89.

40. Fielding RA, Vellas B, Evans WJ, et al. Sarcopenia: an undiagnosed condition in older adults. Current consensus definition: prevalence, etiology, and consequences. International working group on sarcopenia. J Am Med Dir Assoc 2011;12:249-56.

41. Tolea MI, Galvin JE. Sarcopenia and impairment in cognitive and physical performance. Clin Interv Aging 2015;10:663-71.

42. Boyle PA, Buchman AS, Wilson RS, et al. Association of muscle strength with the risk of Alzheimer disease and the rate of cognitive decline in community-dwelling older persons. Arch Neurol 2009;66:1339-44.

43. Kelaiditi E, Canevelli M, Andrieu S, et al. Frailty Index and Cognitive Decline in Alzheimer's Disease: Data from the Impact of Cholinergic Treatment USe Study. J Am Geriatr Soc 2016;64:1165-70.

44. Firth J, Stubbs B, Vancampfort D, et al. Grip Strength Is Associated With Cognitive Performance in Schizophrenia and the General Population: A UK Biobank Study of 476559 Participants. Schizophr Bull 2018;44:728-36.

45. Mavros Y, Gates N, Wilson GC, et al. Mediation of cognitive function improvements by strength gains after resistance training in older adults with mild cognitive impairment: Outcomes of the study of mental and resistance training. J Am Geriatr Soc 2017;65:550-9.
46. Nowson $\mathrm{C}, \mathrm{O}^{\prime}$ Connell S. Protein requirements and recommendations for older people: A review. Nutrients 2015;7:6874-99.

47. Daly RM, O'Connell SL, Mundell NL, et al. Protein-enriched diet, with the use of lean red meat, combined with progressive resistance training enhances lean tissue mass and muscle strength and reduces circulating IL-6 concentrations in elderly women: a cluster randomized controlled trial. Am J Clin Nutr 2014;99:899-910.

48. Morton RW, Murphy KT, McKellar SR, et al. A systematic review, meta-analysis and meta-regression of the effect of protein supplementation on resistance training-induced gains in muscle mass and strength in healthy adults. Br J Sports Med 2018;52:bjsp orts-2017-097608.

49. Mitchell CJ, Milan AM, Mitchell SM, et al. The effects of dietary protein intake on appendicular lean mass and muscle function in elderly men: a 10-wk randomized controlled trial. Am J Clin Nutr 2017;106:1375-83.

50. van de Rest O, van der Zwaluw NL, Tieland M, et al. Effect of resistance-type exercise training with or without protein supplementation on cognitive functioning in frail and pre-frail elderly: secondary analysis of a randomized, double-blind, placebocontrolled trial. Mech Ageing Dev 2014;136-137:85-93.

51. van de Rest O, van der Zwaluw NL, de Groot LCPGM. Literature review on the role of dietary protein and amino acids in cognitive functioning and cognitive decline. Amino Acids 2013;45:1035-45.

52. Chan AW, Tetzlaff JM, Altman DG, et al. SPIRIT 2013 statement: defining standard protocol items for clinical trials. Ann Intern Med 2013;158:200-7.

53. Schulz KF, Altman DG, Moher D. CONSORT Group. CONSORT 2010 statement: updated guidelines for reporting parallel group randomised trials. BMJ 2010;340:c332

54. Boutron I, Moher D, Altman DG, et al. Extending the CONSORT statement to randomized trials of nonpharmacologic treatment: explanation and elaboration. Ann Intern Med 2008;148:295-309.

55. Borg G. Borg's Perceived Exertion and Pain Scales. Human Kinetics,1998 Champaign, IL, US

56. US Department of Health and Human Services. The Report of the Dietary Guidelines Advisory Committee on Dietary Guidelines for Americans. Washington, 2005

57. Nowson CA, McGrath JJ, Ebeling PR, et al. Vitamin D and health in adults in Australia and New Zealand: a position statement. Med J Aust 2012;196:686-7.

58. Yang Y, Breen L, Burd NA, et al. Resistance exercise enhances myofibrillar protein synthesis with graded intakes of whey protein in older men. Br J Nutr 2012;108:1780-8.

59. Yesavage JA. Geriatric Depression Scale. Psychopharmacol Bull 1988;24:709-11.

60. Nasreddine ZS, Phillips NA, Bédirian V, et al. The Montreal Cognitive Assessment, MoCA: a brief screening tool for mild cognitive impairment. J Am Geriatr Soc 2005;53:695-9.

61. Darby DG, Pietrzak RH, Fredrickson J, et al. Intraindividual cognitive decline using a brief computerized cognitive screening test. Alzheimers Dement 2012;8:95-104.

62. Hammers D, Spurgeon E, Ryan K, et al. Reliability of repeated cognitive assessment of dementia using a brief computerized battery. Am J Alzheimers Dis Other Demen 2011;26:326-33.

63. Maruff P, Thomas E, Cysique L, et al. Validity of the CogState brief battery: relationship to standardized tests and sensitivity to cognitive impairment in mild traumatic brain injury, schizophrenia, and AIDS dementia complex. Arch Clin Neuropsychol 2009;24:165-78.

64. Bowie CR, Harvey PD. Administration and interpretation of the Trail Making Test. Nat Protoc 2006;1:2277-81.

65. Sánchez-Cubillo I, Periáñez JA, Adrover-Roig D, et al. Construct validity of the Trail Making Test: role of task-switching, working memory, inhibition/interference control, and visuomotor abilities. J Int Neuropsychol Soc 2009;15:438-50.

66. Giovagnoli AR, Del Pesce M, Mascheroni S, et al. Trail making test: normative values from 287 normal adult controls. Ital J Neurol Sci 1996;17:305-9.

67. Lovibond SH, Lovibond PF. Manual for the Depression Anxiety Stress Scales. Sydney, Australia: Psychology Foundation, 1995.

68. Ware JE, Gandek B. Overview of the SF-36 Health Survey and the International Quality of Life Assessment (IQOLA) Project. J Clin Epidemiol 1998;51:903-12.

69. Hawthorne G, Osborne RH, Taylor A, et al. The SF36 Version 2: critical analyses of population weights, scoring algorithms and population norms. Qual Life Res 2007;16:661-73.

70. Buysse DJ, Reynolds CF, Monk TH, et al. The pittsburgh sleep quality index: A new instrument for psychiatric practice and research Psychiatry Res 1989;28:193-213

71. Daly RM, Miller EG, Dunstan DW, et al. The effects of progressive resistance training combined with a whey-protein drink and vitamin 
D supplementation on glycaemic control, body composition and cardiometabolic risk factors in older adults with type 2 diabetes: study protocol for a randomized controlled trial. Trials 2014;15:431.

72. Dite W, Temple VA. A clinical test of stepping and change of direction to identify multiple falling older adults. Arch Phys Med Rehabil 2002;83:1566-71.

73. Podsiadlo D, Richardson S. The timed "Up \& Go": a test of basic functional mobility for frail elderly persons. J Am Geriatr Soc 1991;39:142-8.

74. Jones CJ, Rikli RE, Beam WC. A 30-s chair-stand test as a measure of lower body strength in community-residing older adults. Res $Q$ Exerc Sport 1999;70:113-9.

75. World Health Organization. Waist circumference and waist-hip ratio: Report of a WHO expert consultation. Geneva, 2008. World Health Organization.

76. Nelson H E, Willison J. National Adult Reading Test manual. 2nd ed. Windsor, 1991. NFER-Nelson, Editor.

77. Bright P, Jaldow E, Kopelman MD. The National Adult Reading Test as a measure of premorbid intelligence: a comparison with estimates derived from demographic variables. J Int Neuropsychol Soc 2002;8:847-54.

78. Walsh SP, Raman R, Jones KB, et al. ADCS Prevention Instrument Project: the Mail-In Cognitive Function Screening Instrument (MCFSI). Alzheimer Dis Assoc Disord 2006;20(4 Suppl 3):S170-S178.

79. Amariglio RE, Donohue MC, Marshall GA, et al. Tracking early decline in cognitive function in older individuals at risk for Alzheimer disease dementia: the Alzheimer's Disease Cooperative Study Cognitive Function Instrument. JAMA Neurol 2015;72:446-54

80. McCrae RR, Costa PTJ. NEO Inventories: Professional manual. Lutz, FL: Psychological Assessment Resources, Inc., 2010.

81. Slavin MJ, Brodaty $\mathrm{H}$, Kochan NA, et al. Prevalence and predictors of "subjective cognitive complaints" in the Sydney Memory and Ageing Study. Am J Geriatr Psychiatry 2010;18:701-10.

82. Lepage G, Roy CC. Improved recovery of fatty acid through direct transesterification without prior extraction or purification. J Lipid Res $1984 ; 25: 1391-6$
83. Smith JD. Apolipoproteins and aging: emerging mechanisms. Ageing Res Rev 2002;1:345-65.

84. Bennet AM, Di Angelantonio E, Ye Z, et al. Association of apolipoprotein $\mathrm{E}$ genotypes with lipid levels and coronary risk. JAMA 2007;298:1300-11.

85. Corder EH, Saunders AM, Strittmatter WJ, et al. Gene dose of apolipoprotein E type 4 allele and the risk of Alzheimer's disease in late onset families. Science 1993;261:921-3.

86. Solomon A, Turunen $\mathrm{H}, \mathrm{Ngandu} \mathrm{T}$, et al. Effect of the apolipoprotein e genotype on cognitive change during a multidomain lifestyle intervention: A subgroup analysis of a randomized clinical trial. JAMA Neurol 2018;75:462-70

87. Stewart AL, Mills KM, King AC, et al. CHAMPS physical activity questionnaire for older adults: outcomes for interventions. $\mathrm{Med} \mathrm{Sci}$ Sports Exerc 2001;33:1126-41.

88. Swierk M, Williams PG, Wilcox J, et al. Validation of an Australian electronic food frequency questionnaire to measure polyunsaturated fatty acid intake. Nutrition 2011;27:641-6.

89. GBD 2015 Neurological Disorders Collaborator Group. Global, regional, and national burden of neurological disorders during 19902015: a systematic analysis for the Global Burden of Disease Study 2015. Lancet Neurol 2017;16:877-97.

90. Ngandu T, Lehtisalo J, Solomon A, et al. A 2 year multidomain intervention of diet, exercise, cognitive training, and vascular risk monitoring versus control to prevent cognitive decline in at-risk elderly people (FINGER): a randomised controlled trial. Lancet 2015;385:2255-63.

91. Montero-Odasso M, Almeida QJ, Burhan AM, et al. SYNERGIC TRIAL (SYNchronizing Exercises, Remedies in Gait and Cognition) a multi-Centre randomized controlled double blind trial to improve gait and cognition in mild cognitive impairment. BMC Geriatr 2018;18:93.

92. Lampit A, Valenzuela M. Pointing the FINGER at multimodal dementia prevention. The Lancet 2015;386:1625-6. 